TRANSACTIONS OF THE

AMERICAN MATHEMATICAL SOCIETY

Volume 361, Number 2, February 2009, Pages 1039-1052

S 0002-9947(08)04547-9

Article electronically published on August 18, 2008

\title{
TOEPLITZ OPERATORS AND LOCALIZATION OPERATORS
}

\author{
MIROSLAV ENGLIŠ
}

\begin{abstract}
We show that for any localization operator on the Fock space with polynomial window, there exists a constant coefficient linear partial differential operator $D$ such that the localization operator with symbol $f$ coincides with the Toeplitz operator with symbol $D f$. An analogous result also holds in the context of Bergman spaces on bounded symmetric domains. This verifies a recent conjecture of Coburn and simplifies and generalizes recent results of Lo.
\end{abstract}

\section{INTRODUCTION}

Let $\mathcal{F}$ be the Fock, or Segal-Bargmann, space of all entire functions on $\mathbf{C}^{n}$ square-integrable with respect to the Gaussian

$$
d \mu(z):=e^{-\|z\|^{2} / 2} \frac{d z}{(2 \pi)^{n}},
$$

$d z$ being the Lebesgue volume measure on $\mathbf{C}^{n}$. It is well known (and easy to check) that the Weyl operators

$$
W_{a} f(z):=e^{\langle z, a\rangle / 2-\|a\|^{2} / 4} f(z-a), \quad a \in \mathbf{C}^{n},
$$

are unitary on $L^{2}\left(\mathbf{C}^{n}, d \mu\right)$ and on $\mathcal{F}$. For $w \in \mathcal{F}$ and $f \in L^{\infty}\left(\mathbf{C}^{n}\right)$, the GaborDaubechies localization operator $L_{f}^{(w)}$ with "window" $w$ and "symbol" $f$ is the operator on $\mathcal{F}$ defined by

$$
\left\langle L_{f}^{(w)} u, v\right\rangle=(2 \pi)^{-n} \int_{\mathbf{C}^{n}} f(a)\left\langle u, W_{a} w\right\rangle\left\langle W_{a} w, v\right\rangle d a, \quad u, v \in \mathcal{F} .
$$

On the other hand, for $f \in L^{\infty}\left(\mathbf{C}^{n}\right)$, the Toeplitz operator $T_{f}$ with symbol $f$ is the operator on $\mathcal{F}$ defined by

$$
T_{f} u=P(f u), \quad u \in \mathcal{F},
$$

where $P: L^{2}\left(\mathbf{C}^{n}, d \mu\right) \rightarrow \mathcal{F}$ is the orthogonal projection. Using the fact that the exponentials

$$
K_{y}(z):=K(z, y):=e^{\langle z, y\rangle / 2}
$$

serve as the reproducing kernel for $\mathcal{F}$, in the sense that

$$
f(x)=\left\langle f, K_{x}\right\rangle=\int_{\mathbf{C}^{n}} f(y) K(x, y) d \mu(y) \quad \forall f \in \mathcal{F}, \forall x \in \mathbf{C}^{n},
$$

Received by the editors July 31, 2006 and, in revised form, May 7, 2007.

2000 Mathematics Subject Classification. Primary 47B35; Secondary 42C40, 32M15, 81R30.

Key words and phrases. Toeplitz operator, localization operator, bounded symmetric domain, Segal-Bargmann space, Bergman space.

This research was supported by GA ČR grant no. 201/06/0128 and Ministry of Education research plan no. MSM4781305904.

(C) 2008 American Mathematical Society Reverts to public domain 28 years from publication 
we can also express $T_{f}$ as an integral operator

$$
T_{f} u(x)=\int_{\mathbf{C}^{n}} f(y) u(y) K(x, y) d \mu(y), \quad u \in \mathcal{F}, x \in \mathbf{C}^{n} .
$$

It is immediate from (3) that for $f \in L^{\infty}\left(\mathbf{C}^{n}\right), T_{f}$ is bounded and

$$
\left\|T_{f}\right\| \leq\|f\|_{\infty} .
$$

In principle, it is possible to define $T_{f}$ by the formula (3) or (4) even for some unbounded symbols $f$ : for instance, for all $f$ such that $f K_{y} \in L^{2}\left(\mathbf{C}^{n}, d \mu\right)$ for all $y \in \mathbf{C}^{n}$. Then $T_{f}$ is a densely defined, closed operator on $\mathcal{F}$. Similarly, (2) can be extended also to some unbounded symbols $f$ as a densely defined operator.

It was observed by Coburn ([C2, C3 $)$ that for $w=\mathbf{1}$,

$$
L_{f}^{(w)}=T_{f}
$$

for all $f \in L^{\infty}\left(\mathbf{C}^{n}\right)$, while for $w(z)=2^{-1 / 2} z_{1}$ and $w(z)=2^{-3 / 2} z_{1}^{2}$, respectively,

$$
\begin{aligned}
& L_{f}^{(w)}=T_{f+2 \partial_{1} \bar{\partial}_{1} f}, \\
& L_{f}^{(w)}=T_{f+4 \partial_{1} \bar{\partial}_{1} f+2\left(\partial_{1} \bar{\partial}_{1}\right)^{2} f},
\end{aligned}
$$

for any $f$ which is either a polynomial in $z, \bar{z}$ or belongs to the algebra $B_{a}\left(\mathbf{C}^{n}\right)$ of Fourier-Stieltjes transforms of compactly supported complex measures on $\mathbf{C}^{n}$. (Here $\partial_{1}=\partial / \partial z_{1}$ and $\bar{\partial}_{1}=\partial / \partial \bar{z}_{1}$.) This allows the amalgamation of the substantial work already done in studying $T_{f}([\mathrm{Be},[\mathrm{BC} 1,[\mathrm{BC} 2,[\mathrm{BC}],[\mathrm{C} 1$, ]Ja, $[\mathrm{Zh}])$ and $\left.\left.L_{f}^{(w)}([\overline{\mathrm{D} 1}], \overline{\mathrm{D} 2}], \overline{\mathrm{FN}}\right], \overline{\mathrm{WO}}\right)$. Coburn's most general result was that for any polynomial $w \in \mathcal{F}$ there exists a linear partial differential operator $D=D^{(w)}$, whose coefficients are polynomials in $z$ and $\bar{z}$, such that

$$
L_{f}^{(w)} p=T_{D f} p
$$

for any polynomial $p \in \mathcal{F}$ and any polynomial $f$ in $z$ and $\bar{z}$. He also conjectured that $D$ was actually a constant coefficient linear differential operator and (6) held also for all $f \in B_{a}\left(\mathbf{C}^{n}\right)$. This conjecture was verified by M.-L. Lo [Lo], who showed that (6) holds for any polynomials $p, w \in \mathcal{F}$ and any $f \in E\left(\mathbf{C}^{n}\right)$, where

$$
\begin{gathered}
E\left(\mathbf{C}^{n}\right):=\left\{f \in C^{\infty}\left(\mathbf{C}^{n}\right): \text { for any multiindex } k \text {, there exist } M, \alpha>0\right. \\
\text { such that } \left.\left|D^{k} f(z)\right| \leq M e^{\alpha\|z\|} \forall z \in \mathbf{C}^{n}\right\}
\end{gathered}
$$

contains both $B_{a}\left(\mathbf{C}^{n}\right)$ and all polynomials in $z$ and $\bar{z}$.

Lo's proof went by a brute-force computation to establish the result for polynomials $f$ (in $z$ and $\bar{z}$ ), and then an approximation argument was used to extend it to all $f \in E\left(\mathbf{C}^{n}\right)$.

In this note, we present a simpler proof of these results, which also yields a bit more precise information for "nicer" symbols $f$.

Theorem 1. For any polynomial $w \in \mathcal{F}$, there exists a constant coefficient linear partial differential operator $D=D^{(w)}$ such that for any $f \in B C^{\infty}\left(\mathbf{C}^{n}\right)$ (the space of all $C^{\infty}$ functions on $\mathbf{C}^{n}$ whose partial derivatives of all orders are bounded),

$$
L_{f}^{(w)}=T_{D f} \quad \text { on } \mathcal{F} .
$$


Explicitly, the operator $D$ is given by

$$
D^{(w)}=\left[e^{\Delta / 2}|w(z)|^{2}\right]_{\substack{z \mapsto-2 \bar{\partial} \\ \bar{z} \mapsto-2 \partial}} \cdot
$$

Here $e^{\Delta / 2}$ should be understood as the infinite series

$$
e^{\Delta / 2}=\sum_{k=0}^{\infty} \frac{\Delta^{k}}{k ! 2^{k}} .
$$

This infinite sum makes sense since, as $w$ is assumed to be a polynomial, $\Delta^{k}|w|^{2}$ vanishes as soon as $k>\operatorname{deg} w$; thus there are only finitely many nonzero terms. Note also that for $f \in B C^{\infty}$ both sides of (8) are bounded operators, so the validity is not restricted to polynomials $p$ as in (6). In fact, the left-hand side in (8) is a bounded operator for any $f \in L^{\infty}\left(\mathbf{C}^{n}\right)$ (see Proposition 2), so (8) tells us that Toeplitz operators can even be defined and nice (i.e. bounded) for the fairly wild symbols $D f, f \in L^{\infty}$ (which are distributions at best).

One more virtue of our proof is that it uses solely harmonic analysis methods, and thus easily extends also to other situations than the Segal-Bargmann space on $\mathbf{C}^{n}$, for instance, to the standard weighted Bergman spaces on bounded symmetric domains, thus making contact with the work of Arazy and Upmeier [AU], de Mari and Nowak $[\mathrm{MN}]$, and others.

The paper is organized as follows. In Section 2, we review some preliminaries from Segal-Bargmann analysis. In Section 3 , Theorem 1 is proved, and also extended to a wider class of functions $f$ (including the polynomials, the algebra $B_{a}\left(\mathbf{C}^{n}\right)$, and the space $E\left(\mathbf{C}^{n}\right)$ from (77) $)$. Generalizations to bounded symmetric domains are described in the final Section 4 .

A substantial portion of this work was done during the Finnish Mathematical Society Visitor Program 2005-2006 in Helsinki and Joensuu; the author expresses his gratitude for the support and the nice time for research he had there.

\section{BEREZIN SYMBOLS}

In addition to $K_{a}$, we also consider the normalized reproducing kernels

$$
k_{a}(z):=\frac{K_{a}(z)}{\left\|K_{a}\right\|}=e^{\langle z, a\rangle / 2-\|a\|^{2} / 4} .
$$

Note that the Weyl operators (1) can then be written simply as

$$
W_{a} f(z)=k_{a}(z) f(z-a) .
$$

In particular, as $k_{0}=\mathbf{1}$ (the function constant one),

$$
k_{a}=W_{a} \mathbf{1}, \quad \forall a \in \mathbf{C}^{n} .
$$

One checks easily that $W_{a}$ satisfy the composition law

$$
W_{a} W_{b}=e^{(\bar{a} b-a \bar{b}) / 4} W_{a+b}, \quad \forall a, b \in \mathbf{C}^{n} .
$$

Consequently, $W_{a}^{*}=W_{-a}$ and

$$
\begin{aligned}
W_{a} k_{b} & =e^{(\bar{a} b-a \bar{b}) / 4} k_{a+b}, \\
W_{a}^{*} k_{b} & =e^{(a \bar{b}-\bar{a} b) / 4} k_{b-a} .
\end{aligned}
$$


In particular, for $w=1$, we get for any $u, v \in \mathcal{F}$,

$$
\begin{aligned}
\left\langle L_{f}^{(\mathbf{1})} u, v\right\rangle & =(2 \pi)^{-n} \int_{\mathbf{C}^{n}} f(a)\left\langle u, k_{a}\right\rangle\left\langle k_{a}, v\right\rangle d a \\
& =\int_{\mathbf{C}^{n}} f(a)\left\langle u, K_{a}\right\rangle\left\langle K_{a}, v\right\rangle d \mu(a) \\
& =\int_{\mathbf{C}^{n}} f(a) u(a) \overline{v(a)} d \mu(a) \\
& =\langle f u, v\rangle \\
& =\left\langle T_{f} u, v\right\rangle
\end{aligned}
$$

so that indeed

$$
L_{f}^{(\mathbf{1})}=T_{f} .
$$

The next proposition is thus an analogue of (5) for an arbitrary window $w$. An analogous assertion is valid even in the much more general context of any squareintegrable irreducible unitary representation of a unimodular group; see for instance Wong [Wo, Proposition 12.2], or [E] for an even further generalization; in the very special case that we have here, it is possible to give a simple direct proof based on the Fourier transform.

Proposition 2. For any $w \in \mathcal{F}$ and $f \in L^{\infty}\left(\mathbf{C}^{n}\right)$, the localization operator $L_{f}^{(w)}$ is bounded, and

$$
\left\|L_{f}^{(w)}\right\| \leq\|f\|_{\infty}\|w\|^{2} .
$$

Proof. It is more convenient to pass from $\mathcal{F}$ to $L^{2}\left(\mathbf{R}^{n}\right)$, via the Bargmann transform

$$
\beta f(z):=c_{n} \int_{\mathbf{R}^{n}} f(x) e^{x z-x^{2} / 2-z^{2} / 4} d x .
$$

With the proper choice of the constant $c_{n}$, this is a unitary isomorphism of $L^{2}\left(\mathbf{R}^{n}\right)$ onto $\mathcal{F}$; see e.g. Folland $\left[\mathrm{Fo}\right.$. (Here $x^{2}=x_{1}^{2}+\cdots+x_{n}^{2}$ for $x \in \mathbf{R}^{n}$, and similarly for $x z$ and $z^{2}$.) Its inverse is given by

$$
\beta^{-1} F(x)=c_{n}^{\prime} \int_{\mathbf{C}^{n}} F(z) e^{x \bar{z}-x^{2} / 2-\bar{z}^{2} / 4} e^{-\|z\|^{2} / 2} d z,
$$

and the Weyl operators (1) satisfy $W_{u+i v}=\beta U_{u, v} \beta^{-1}$, where the unitary operators $U_{u, v}$ on $L^{2}\left(\mathbf{R}^{n}\right)$ are given by

$$
U_{u, v} f(x)=e^{i u v / 2-i v x} f(x-u), \quad x, u, v \in \mathbf{R}^{n} .
$$

It follows that

$$
\beta^{-1} L_{f}^{(w)} \beta=(2 \pi)^{-n} \int_{\mathbf{R}^{n}} \int_{\mathbf{R}^{n}} f(u+i v)\left\langle\cdot, U_{u, v} H\right\rangle\left\langle U_{u, v} H, \cdot\right\rangle d u d v,
$$

where $H=\beta^{-1} w$. To prove the proposition, it therefore suffices to show that

$$
\left|(2 \pi)^{-n} \int_{\mathbf{R}^{n}} \int_{\mathbf{R}^{n}} f(u+i v)\left\langle F, U_{u, v} H\right\rangle\left\langle U_{u, v} H, G\right\rangle d u d v\right| \leq\|f\|_{\infty}\|H\|^{2}\|F\|\|G\|
$$

for all $F, G \in L^{2}\left(\mathbf{R}^{n}\right)$. 
By the Cauchy-Schwarz inequality, the left-hand side is bounded by

$$
(2 \pi)^{-n}\|f\|_{\infty}\left(\int_{\mathbf{R}^{n}} \int_{\mathbf{R}^{n}}\left|\left\langle F, U_{u, v} H\right\rangle\right|^{2} d u d v\right)^{1 / 2}\left(\int_{\mathbf{R}^{n}} \int_{\mathbf{R}^{n}}\left|\left\langle G, U_{u, v} H\right\rangle\right|^{2} d u d v\right)^{1 / 2} .
$$

It is therefore enough to prove that

$$
(2 \pi)^{-n} \int_{\mathbf{R}^{n}} \int_{\mathbf{R}^{n}}\left|\left\langle F, U_{u, v} H\right\rangle\right|^{2} d u d v \leq\|F\|^{2}\|H\|^{2}
$$

for any $F, H \in L^{2}\left(\mathbf{R}^{n}\right)$. However,

$$
\left\langle F, U_{u, v} H\right\rangle=\int_{\mathbf{R}^{n}} F(x) e^{-i u v / 2} e^{i v x} \overline{H(x-u)} d x=(2 \pi)^{n / 2} e^{-i u v / 2} \hat{h}_{u}(v),
$$

where $\hat{h}_{u}$ is the Fourier transform of the function $h_{u}(x)=F(x) \overline{H(x-u)}$. Thus, by Parseval,

$$
\begin{aligned}
(2 \pi)^{-n} \int_{\mathbf{R}^{n}} & \int_{\mathbf{R}^{n}}\left|\left\langle F, U_{u, v} H\right\rangle\right|^{2} d u d v \\
& =\int_{\mathbf{R}^{n}} \int_{\mathbf{R}^{n}}\left|\hat{h}_{u}(v)\right|^{2} d u d v \\
& =\int_{\mathbf{R}^{n}} \int_{\mathbf{R}^{n}}\left|h_{u}(x)\right|^{2} d u d x \\
& =\int_{\mathbf{R}^{n}} \int_{\mathbf{R}^{n}}|F(x)|^{2}|H(x-u)|^{2} d u d x \\
& =\int_{\mathbf{R}^{n}}|F(x)|^{2}\left[\int_{\mathbf{R}^{n}}|H(x-u)|^{2} d u\right] d x \\
& =\int_{\mathbf{R}^{n}}|F(x)|^{2}\left[\int_{\mathbf{R}^{n}}|H(y)|^{2} d y\right] d x \quad(y:=x-u) \\
& =\|F\|^{2}\|H\|^{2} .
\end{aligned}
$$

Remark. We see that we have in fact an equality in (14). On the general level of square-integrable irreducible representations of an arbitrary unimodular group, this is of course just an immediate consequence of the Schur orthogonality relations.

Recall that for a bounded linear operator $T$ on $\mathcal{F}$, the Berezin symbol of $T$ is the function $\widetilde{T}$ on $\mathbf{C}^{n}$ defined by

$$
\widetilde{T}(x):=\left\langle T k_{x}, k_{x}\right\rangle .
$$

Again, the definition makes sense even for unbounded operators, as long as the reproducing kernels $k_{x}$ are in the domain of $T$, for all $x$. The following proposition records some properties of the Berezin symbol which we will need.

Proposition 3. (a) The function $\widetilde{T}$ is real-analytic;

(b) $\widetilde{T}$ vanishes identically only if $T=0$; 
(c) $\|\widetilde{T}\|_{\infty} \leq\|T\| ;$

(d) for any $a \in \mathbf{C}^{n}$,

$$
\left(W_{a}^{*} T W_{a}\right)^{\sim}=\widetilde{T}(\cdot+a) .
$$

Proof. All this is well known, but here is the proof for completeness. Note that $\widetilde{T}(x)$ is the restriction to the diagonal $x=y$ of the function

$$
\begin{aligned}
\frac{\left\langle T K_{y}, K_{x}\right\rangle}{\left\langle K_{y}, K_{x}\right\rangle}=e^{-\langle x, y\rangle / 2}\left\langle T e^{\langle\cdot, y\rangle / 2}, e^{\langle\cdot, x\rangle / 2}\right\rangle & =e^{-\langle x, y\rangle / 2}\left(T e^{\langle\cdot, y\rangle / 2}\right)(x) \\
& =e^{-\langle x, y\rangle / 2} \overline{\left(T^{*} e^{\langle\cdot, x\rangle / 2}\right)(y)},
\end{aligned}
$$

which is holomorphic in $x$ and $\bar{y}$; in particular, $\widetilde{T}$ is a real-analytic function. Further, it is known that such functions are uniquely determined by their restriction to the diagonal (see e.g. Folland [Fo, Proposition 1.69); hence $\widetilde{T} \equiv 0$ only if $\left\langle T K_{y}, K_{x}\right\rangle=$ $T K_{y}(x)=0 \forall x, y$, which implies that $T=0$ since the linear combinations of $K_{y}$, $y \in \mathbf{C}^{n}$, are dense in $\mathcal{F}$. Finally, (c) is immediate from the Schwarz inequality, and the covariance property (15) is immediate from (12).

\section{MAIN RESULTS}

Proof of Theorem 1. From the definition of the localization operators $L_{F}^{(w)}$, we have for any $c \in \mathbf{C}^{n}$,

$$
\begin{aligned}
L_{f(\cdot+c)}^{(w)} & =(2 \pi)^{-n} \int f(a+c)\left\langle\cdot, W_{a} w\right\rangle W_{a} w d a \\
& =(2 \pi)^{-n} \int f(x)\left\langle\cdot, W_{x-c} w\right\rangle W_{x-c} w d x \\
& =W_{c}^{*} L_{f}^{(w)} W_{c}
\end{aligned}
$$

by (11). In particular, for $w=1$, we get by (13),

$$
T_{f(\cdot+c)}=W_{c}^{*} T_{f} W_{c}
$$

By Proposition 2, and parts (a), (c) and (d) of Proposition 3, we thus see that the two maps

$$
f \mapsto \widetilde{L_{f}^{(w)}}, \quad f \mapsto \widetilde{T_{f}}
$$

both map $L^{\infty}\left(\mathbf{C}^{n}\right)$ continuously into bounded real-analytic functions on $\mathbf{C}^{n}$, and commute with translations. Recall now (see e.g. $[\mathrm{Ru}$, Theorem 6.33) that for any continuous linear map $V$ from $\mathcal{D}\left(\mathbf{C}^{n}\right)$ into $C\left(\mathbf{C}^{n}\right)$ which commutes with translations there is a unique distribution $v \in \mathcal{D}^{\prime}\left(\mathbf{C}^{n}\right)$ such that $V f=v * f$ for all $f \in \mathcal{D}$. Thus there exist distributions $k=k^{(w)}$ and $h=k^{(\mathbf{1})}$ on $\mathbf{C}^{n}$ such that

$$
\begin{aligned}
\widetilde{L_{f}^{(w)}} & =k * f, \\
\widetilde{T_{f}} & =h * f,
\end{aligned}
$$


for all $f \in \mathcal{D}\left(\mathbf{C}^{n}\right)$. To find what $k$ and $h$ are, note that for any $f \in L^{\infty}\left(\mathbf{C}^{n}\right)$ and $z \in \mathbf{C}^{n}$,

$$
\begin{aligned}
\widetilde{L_{f}^{(w)}}(z) & =\left\langle L_{f}^{(w)} k_{z}, k_{z}\right\rangle \\
& =(2 \pi)^{-n} \int f(a)\left\langle k_{z}, W_{a} w\right\rangle\left\langle W_{a} w, k_{z}\right\rangle d a \\
& =(2 \pi)^{-n} \int f(a)\left|\left\langle W_{a}^{*} k_{z}, w\right\rangle\right|^{2} d a \\
& =(2 \pi)^{-n} \int f(a)\left|\left\langle k_{z-a}, w\right\rangle\right|^{2} d a \quad \text { by (12) } \\
& =(2 \pi)^{-n} \int f(z-y)\left|\left\langle k_{y}, w\right\rangle\right|^{2} d y \\
& =(2 \pi)^{-n} \int f(z-y)\left|\left\langle K_{y}, w\right\rangle\right|^{2} e^{-\|y\|^{2} / 2} d y \\
& =(2 \pi)^{-n} \int f(z-y)|w(y)|^{2} e^{-\|y\|^{2} / 2} d y \\
& =\left(f *(2 \pi)^{-n}|w|^{2} e^{-\|\cdot\|^{2} / 2}\right)(z) .
\end{aligned}
$$

Thus $k$ is not only a distribution but a function, given by

$$
k(z)=(2 \pi)^{-n}|w(z)|^{2} e^{-\|z\|^{2} / 2},
$$

and, taking $w=\mathbf{1}$,

$$
h(z)=(2 \pi)^{-n} e^{-\|z\|^{2} / 2} .
$$

It also follows from the last computation that (17) holds not only for $f \in \mathcal{D}\left(\mathbf{C}^{n}\right)$, but for any $f \in L^{\infty}\left(\mathbf{C}^{n}\right)$.

Observe now that for any multiindices $j, k$, the Leibniz formula implies that

$$
\partial^{j} \bar{\partial}^{k} e^{-\|z\|^{2} / 2}=e^{-\|z\|^{2} / 2}\left[\left(-\frac{1}{2}\right)^{|j+k|} \bar{z}^{j} z^{k}+\text { lower order terms }\right] .
$$

By a straightforward induction argument, it follows that there exists a unique differential operator $D=D^{(w)}$ with constant coefficients such that

$$
D e^{-\|\cdot\|^{2} / 2}=|w|^{2} e^{-\|\cdot\|^{2} / 2},
$$

i.e. $D h=k$. By the properties of convolution,

$$
h * D f=D h * f=k * f
$$

for any reasonable $f$ (for instance, whenever all derivatives of $f$ up to the order of $D$ are bounded). Consequently,

$$
\widetilde{T_{D f}}=h * D f=k * f=\widetilde{L_{f}^{(w)}}
$$

for any $f \in B C^{\infty}\left(\mathbf{C}^{n}\right)$. By part (b) of Proposition 3, this implies that

$$
T_{D f}=L_{f}^{(w)}
$$

thus completing the proof of (8) . 
It remains to show that the operator $D$ is given by the formula (9). To this end, write out the "lower order terms" in (20) explicitly:

$$
\begin{aligned}
\partial^{j} \bar{\partial}^{k} e^{-\|z\|^{2} / 2} & =\partial^{j}\left[\left(-\frac{z}{2}\right)^{k} e^{-\|z\|^{2} / 2}\right] \\
& =\sum_{l \subset j}\left(\begin{array}{l}
j \\
l
\end{array}\right)\left(-\frac{1}{2}\right)^{|k|} \frac{k !}{(k-l) !} z^{k-l}\left(-\frac{\bar{z}}{2}\right)^{j-l} e^{-\|z\|^{2} / 2} \\
& =\sum_{l} \frac{j !}{(j-l) !} \bar{z}^{j-l} \frac{k !}{(k-l) !} z^{k-l}\left(-\frac{1}{2}\right)^{|j+k-l|} \frac{e^{-\|z\|^{2} / 2}}{l !} \\
& =\left(-\frac{1}{2}\right)^{|j+k|} e^{-\|z\|^{2} / 2} \sum_{l}\left(\bar{\partial}^{l} \bar{z}^{j}\right) \cdot\left(\partial^{l} z^{k}\right) \frac{(-2)^{|l|}}{l !} \\
& =\left(-\frac{1}{2}\right)^{|j+k|} e^{-\|z\|^{2} / 2} \sum_{L=0}^{\infty} \frac{(-2)^{L}}{L !} \sum_{|l|=L}\left(\begin{array}{l}
L \\
l
\end{array}\right) \partial^{l} \bar{\partial}^{l} \bar{z}^{j} z^{k} \\
& =\left(-\frac{1}{2}\right)^{|j+k|} e^{-\|z\|^{2} / 2} \sum_{L=0}^{\infty} \frac{(-2)^{L}}{L !}\left(\frac{\Delta}{4}\right)^{L} \bar{z}^{j} z^{k} \\
& =\left(-\frac{1}{2}\right)^{|j+k|} e^{-\|z\|^{2} / 2} e^{-\Delta / 2} \bar{z}^{j} z^{k} .
\end{aligned}
$$

It follows that for any polynomial $p$ in two variables with complex coefficients,

$$
p(-2 \partial,-2 \bar{\partial}) e^{-\|z\|^{2} / 2}=e^{-\|z\|^{2} / 2} e^{-\Delta / 2} p(\bar{z}, z) .
$$

Thus if we choose

$$
p(\bar{z}, z)=e^{\Delta / 2}|w(z)|^{2},
$$

then $p(-2 \partial,-2 \bar{\partial})=D$. This completes the proof of Theorem 1

Corollary 4. Let $w_{1}, w_{2} \in \mathcal{F}$ be polynomials. Then the following two assertions are equivalent:

(a) There exists a constant coefficient linear differential operator D such that

$$
L_{f}^{\left(w_{2}\right)}=L_{D f}^{\left(w_{1}\right)}
$$

for all $f \in \mathcal{D}\left(\mathbf{C}^{n}\right)$.

(b) The polynomial $e^{\Delta / 2}\left|w_{2}\right|^{2}$ is divisible by the polynomial $e^{\Delta / 2}\left|w_{1}\right|^{2}$.

Further, if (a) or (b) are fulfilled, then $D$ is of order $2\left(\operatorname{deg} w_{2}-\operatorname{deg} w_{1}\right)$ and (22) holds for all $f \in B C^{\infty}\left(\mathbf{C}^{n}\right)$.

Proof. This is immediate from (8) and (9).

Note that we have proved (8) not only for $f \in B C^{\infty}$, but in fact for any $f \in L^{\infty}$ whose derivatives up to the order of $D$ are bounded. Going through the above arguments with some care, it is not difficult to extend this even further. Let $r$ be the degree of $w$ and denote

$$
\begin{gathered}
\mathcal{M}_{r}:=\left\{f \in C^{2 r}\left(\mathbf{C}^{n}\right): \text { for any multiindices } j, k \text { with }|j|,|k| \leq r\right. \\
\text { and any } \left.a>0, e^{a\|\cdot\|}\left|\partial^{j} \bar{\partial}^{k} f\right| e^{-\|\cdot\|^{2} / 2} \in L^{\infty}\left(\mathbf{C}^{n}\right)\right\} .
\end{gathered}
$$


Observe that the condition implies that for any $m \geq 0$ and $|j|,|k| \leq r,\|z\|^{m}\left|\partial^{j} \bar{\partial}^{k} f\right|$. $e^{-\|z\|^{2} / 2}$ belongs to $L^{1}$ and vanishes at infinity. Integrating by parts in

$$
\int f(z-x) D e^{-\|x\|^{2} / 2} d x
$$

it therefore follows that

$$
f * D h=D f * h \quad \forall f \in \mathcal{M}_{r}
$$

i.e., (21) still holds for $f \in \mathcal{M}_{r}$. Thus again

$$
\widetilde{T_{D f}}=\widetilde{L_{f}^{(w)}} .
$$

Since now $T_{D f}$ and $L_{f}^{(w)}$ need no longer be bounded in general, it is not clear whether this implies $T_{D f}=L_{f}^{(w)}$; however, from the proof of part (b) of Proposition 3 it is clear at least that $T_{D f} K_{z}=L_{f}^{(w)} K_{z}$ for any $z \in \mathbf{C}^{n}$. Thus we arrive at the following strengthening of Theorem 1 .

Theorem 5. Let $w \in \mathcal{F}$ be a polynomial of degree $r$, and let $\mathcal{M}_{r}$ be as in (23). Then for any $f \in \mathcal{M}_{r}, T_{D f}$ and $L_{f}^{(w)}$ coincide on the linear span of $K_{z}, z \in \mathbf{C}^{n}$.

Note that $E\left(\mathbf{C}^{n}\right) \subset \mathcal{M}_{r}$ for any $r$; thus, in particular, the last theorem covers completely the main result of [L] (except that the polynomials $p$ are replaced by linear combinations of $K_{z}$ ).

We conclude this section by a generalization in a different direction. It may seem a little artificial at first sight, but becomes very natural after we pass to the bounded symmetric domains in the next section. For any bounded linear operator $A$ on $\mathcal{F}$, we may define a "localization operator" with symbol $f$ and "window" $A$ by

$$
L_{f}^{(A)}:=(2 \pi)^{-n} \int_{\mathbf{C}^{n}} f(a) W_{a} A W_{a}^{*} d a .
$$

The localization operators $L_{f}^{(w)}$ considered so far are recovered upon choosing $A=$ $\langle\cdot, w\rangle w$.

We then have the following generalizations of Proposition 2 and Theorem 1

Proposition 6. If $A$ is trace-class, then the integral (24) converges in the weak operator topology for any $f \in L^{\infty}\left(\mathbf{C}^{n}\right)$, and

$$
\left\|L_{f}^{(A)}\right\| \leq\|f\|_{\infty}\|A\|_{t r}
$$

where $\|\cdot\|_{\text {tr }}$ denotes the trace norm.

Theorem 7. Let $A$ be a finite sum

$$
A=\sum_{j}\left\langle\cdot, u_{j}\right\rangle v_{j}
$$

where $u_{j}, v_{j} \in \mathcal{F}$ are polynomials. Then there exists a unique linear partial differential operator $D=D^{(A)}$ such that

$$
L_{f}^{(A)}=T_{D f} \quad \forall f \in B C^{\infty}\left(\mathbf{C}^{n}\right) .
$$


The proof of Proposition [6 can (again in a much more general setup) be found in [E], or carried out directly along the lines of the proof of Proposition 2, Similarly, Theorem 7 can be proved either by mimicking the proof of Theorem 11, or from Theorem 1 directly using the linearity in $A$ and the familiar polarization identity

$$
\left\langle\cdot, w_{1}\right\rangle w_{2}=\sum_{k=0}^{3} i^{-k}\left\langle\cdot, w_{1}+i^{k} w_{2}\right\rangle\left(w_{1}+i^{k} w_{2}\right) .
$$

\section{BOUNDED SYMMETRIC DOMAINS}

Throughout this section we let $\Omega$ be an irreducible bounded symmetric domain in $\mathbf{C}^{n}$ (i.e. a Cartan domain) in its Harish-Chandra realization (so $\Omega$ is circular with respect to the origin and convex). Let $G$ be the group of all biholomorphic self-maps of $\Omega$; then $G$ acts transitively on $\Omega$, so denoting by $K$ the stabilizer of the origin $0 \in \Omega$ in $G, \Omega$ can be identified with the coset space $G / K$. For each $z \in \Omega$, there exists a unique so-called geodesic symmetry $g_{x} \in G$ interchanging $x$ and the origin, i.e. $g_{x}$ is an involution (that is, $g_{x}=g_{x}^{-1}$ ), $g_{x}(0)=x, g_{x}(x)=0$, and $g_{x}$ has only isolated fixed-points. We refer e.g. to $\mathrm{Ar}], \mathrm{Ko}]$ or $\mathrm{Up}$ for an overview of bounded symmetric domains.

Let $d z$ be the Lebesgue measure on $\Omega$ normalized so that $\Omega$ has total mass one. Abusing the notation a little, we will denote by the same letter $K$ also the Bergman kernel $K_{y}(x)=K(x, y)$ of $\Omega$, i.e. the reproducing kernel of the subspace $\mathcal{H}=$ $L_{\text {hol }}^{2}(\Omega, d z)$ of all holomorphic functions in $L^{2}(\Omega, d z)$. We will also use the same notation $k_{z}=K_{z} /\left\|K_{z}\right\|$ as before for the normalized reproducing kernels.

From the familiar formula for the change of variables, it is immediate that the operators

$$
U_{g}: f \mapsto j_{g^{-1}} \cdot\left(f \circ g^{-1}\right), \quad g \in G,
$$

are unitary on $L^{2}(\Omega)$ and $\mathcal{H}$; here $j_{g}$ denotes the complex Jacobian of the mapping $g$. From the chain rule for derivatives it follows that

$$
U_{g_{1}} U_{g_{2}}=U_{g_{1} g_{2}}, \quad \forall g_{1}, g_{2} \in G,
$$

so that $g \mapsto U_{g}$ is a unitary representation of $G$ in $\mathcal{H}$. In particular, $U_{g}^{*}=U_{g^{-1}}$. From the computation

$$
\begin{aligned}
\left\langle f, U_{g} k_{z}\right\rangle & =\left\langle U_{g^{-1}} f, k_{z}\right\rangle=K(z, z)^{-1 / 2}\left(U_{g^{-1}} f\right)(z) \\
& =K(z, z)^{-1 / 2} j_{g}(z) f(g(z)) \\
& =K(g(z), g(z))^{1 / 2} K(z, z)^{-1 / 2} j_{g}(z)\left\langle f, k_{g(z)}\right\rangle, \quad \forall f \in \mathcal{H},
\end{aligned}
$$

it follows that $U_{g} k_{z}=$ const $\cdot k_{g(z)}$; since $U_{g}$ is unitary and $k_{z}, k_{g(z)}$ are both unit vectors, the constant must be unimodular, i.e.

$$
U_{g} k_{z}=\epsilon_{g, z} k_{g(z)}, \quad\left|\epsilon_{g, z}\right|=1,
$$

which is an analogue of (12).

Yet another consequence of the change-of-variable formula is the equality

$$
K(x, y)=j_{g^{-1}}(x) K\left(g^{-1}(x), g^{-1}(y)\right) \overline{j_{g^{-1}}(y)},
$$

from which it follows that the measure

$$
d \mu(z):=K(z, z) d z, \quad z \in \Omega,
$$

is $G$-invariant. 
Denoting by $d g$ the Haar measure on $G$, we may now define for any bounded linear operator ("window") $A$ on $\mathcal{H}$ and any function ("symbol") $f$ on $G$ the "localization operator"

$$
\mathcal{L}_{f}^{(A)}:=\int_{G} f(g) U_{g} A U_{g}^{*} d g .
$$

Comparing this with (24), we immediately see the drawback that our symbols $f$ now live on $G$, not on $\Omega$. As shown in $\mathrm{AU}$ ] and $[\mathrm{E}$, this can be resolved by restricting to operators $A$ which are $K$-invariant, in the sense that

$$
A U_{k}=U_{k} A \quad \forall k \in K .
$$

Indeed, then for any $g \in G$ we have

$$
U_{g k} A U_{g k}^{*}=U_{g} U_{k} A U_{k}^{*} U_{g}^{*}=U_{g} A U_{g}^{*} .
$$

Thus $U_{g} A U_{g}^{*}$ depends only on the coset $g K$ of $g$ in $G / K$, i.e. only on $g(0) \in \Omega$. We can therefore define unambiguously the operator $A_{z}$, for any $z \in \Omega$, by

$$
A_{z}:=U_{g} A U_{g}^{*} \quad \text { for any } g \in G \text { such that } g(0)=z,
$$

and the localization operator

$$
L_{f}^{(A)}:=\int_{\Omega} f(z) A_{z} d \mu(z) .
$$

Such operator calculi were studied in $[\mathrm{E}$. It was shown there, for instance, that (27) converges in the weak operator topology whenever $f$ is bounded and $A$ is trace-class, and

$$
\left\|L_{f}^{(A)}\right\| \leq\|f\|_{\infty}\|A\|_{\mathrm{tr}}
$$

an analogue of Propositions 2 and 6. Our goal in the rest of this section will be to establish also an analogue of Theorems 1 and 7 . Before stating the latter, we need to review some facts about the structure of $K$-invariant operators.

It is known that under the action $U_{k}$ of the group $K$, the space $\mathcal{H}$ decomposes into an orthogonal direct sum of irreducible subspaces (Peter-Weyl decomposition)

$$
\mathcal{H}=\bigoplus_{\mathbf{m}} \mathcal{P}_{\mathbf{m}}
$$

Here $\mathbf{m}$ ranges over all signatures, i.e. $r$-tuples $\mathbf{m}=\left(m_{1}, \ldots, m_{r}\right)$ of integers satisfying $m_{1} \geq m_{2} \geq \cdots \geq m_{r} \geq 0$; the number $r$ is the $\operatorname{rank}$ of $\Omega$. One has $\mathcal{P}_{(0, \ldots, 0)}=$ $\{$ the constant functions $\}, \mathcal{P}_{(1,0, \ldots, 0)}=\{$ the linear functions $\}$, and, in general, the elements of $\mathcal{P}_{\mathbf{m}}$ are homogeneous polynomials of degree $|\mathbf{m}|:=m_{1}+\cdots+m_{r}$. Let $P_{\mathbf{m}}$ be the orthogonal projection in $\mathcal{H}$ onto $\mathcal{P}_{\mathbf{m}}$. By construction, $P_{\mathbf{m}}$ is a $K$-invariant operator. Conversely, if $A$ is any $K$-invariant operator, then it follows from Schur's lemma that the restriction of $A$ to each $\mathcal{P}_{\mathbf{m}}$ is a multiple of the identity. Thus, the $K$-invariant operators on $\mathcal{H}$ are precisely the operators of the form

$$
A=\sum_{\mathbf{m}} c_{\mathbf{m}} P_{\mathbf{m}}, \quad c_{\mathbf{m}} \in \mathbf{C} .
$$

Clearly $A$ is bounded if and only if $\left\{c_{\mathbf{m}}\right\}$ is a bounded sequence, and $A$ is trace-class if and only if $\sum_{\mathbf{m}} c_{\mathbf{m}} \operatorname{dim} \mathcal{P}_{\mathbf{m}}<\infty$.

The simplest $K$-invariant operator is thus

$$
A=P_{(0, \ldots, 0)}=\langle\cdot, \mathbf{1}\rangle \mathbf{1},
$$


the projection onto the constants. By (26), in that case,

$$
A_{z}=\left\langle\cdot, k_{z}\right\rangle k_{z}
$$

and

$$
\begin{aligned}
L_{f}^{(A)} & =\int_{\Omega} f(z)\left\langle\cdot, k_{z}\right\rangle k_{z} d \mu(z) \\
& =\int_{\Omega} f(z)\left\langle\cdot, K_{z}\right\rangle K_{z} d z \\
& =T_{f}
\end{aligned}
$$

the Toeplitz operator with symbol $f$.

We now have the following analogue of Theorems 1 and 7

Theorem 8. Let $A$ be a $K$-invariant operator on $\mathcal{H}$ of the form

$$
A=\sum_{\text {finite }} c_{\mathbf{m}} P_{\mathbf{m}} \text {. }
$$

Then there exists a unique $G$-invariant linear partial differential operator $D=D^{(A)}$ on $\Omega$ such that

$$
L_{f}^{(A)}=T_{D f} \quad \forall f \in \mathcal{D}(\Omega) .
$$

Proof. The proof is completely parallel to that of Theorem 1 so we will be brief. Using linearity, it is enough to prove the theorem for $A=P_{\mathbf{m}}$, which we will assume from now on. For any bounded linear operator $T$ on $\mathcal{H}$, we again define its Berezin symbol $\widetilde{T}$ by

$$
\widetilde{T}(z)=\left\langle T k_{z}, k_{z}\right\rangle, \quad z \in \Omega .
$$

The proof of Proposition 3 extends to the present setting without any changes, so that again $\|\widetilde{T}\|_{\infty} \leq\|T\|, \widetilde{T}$ is real-analytic, and $\widetilde{T} \equiv 0$ only if $T=0$. By a similar computation as for the Fock space, for any $f \in L^{\infty}(\Omega)$,

$$
\widetilde{L_{f}^{(A)}}(z)=\left\langle L_{f}^{(A)} k_{z}, k_{z}\right\rangle=\int_{\Omega} f(x)\left\langle A_{x} k_{z}, k_{z}\right\rangle d \mu(x) .
$$

Let $g_{x} \in G$ be the geodesic symmetry interchanging $x$ and the origin, so that $g_{x}=g_{x}^{-1}, g_{x}(0)=x$ and $g_{x}(x)=0$. Then $\left\langle A_{x} k_{z}, k_{z}\right\rangle=\left\langle A U_{g_{x}}^{*} k_{z}, U_{g_{x}}^{*} k_{z}\right\rangle=$ $\left\langle A k_{g_{x}(z)}, k_{g_{x}(z)}\right\rangle$, by (26). Since $g_{x}\left(g_{z}(0)\right)=g_{x}(z)=g_{g_{x}(z)}(0)$, there exists $k \in K$ such that $g_{x} g_{z}=g_{g_{x}(z)} k$; taking inverses gives $k g_{z} g_{x}=g_{g_{x}(z)}$, whence $g_{x}(z)=$ $g_{g_{x}(z)}(0)=k\left(g_{z}\left(g_{x}(0)\right)\right)=k\left(g_{z}(x)\right)$. As $A$ is $K$-invariant, $\left\langle A k_{g_{x}(z)}, k_{g_{x}(z)}\right\rangle=$ $\left\langle A U_{k} k_{g_{z}(x)}, U_{k} k_{g_{z}(x)}\right\rangle=\left\langle A k_{g_{z}(x)}, k_{g_{z}(x)}\right\rangle=\widetilde{A}\left(g_{z}(x)\right)$. Thus

$$
\widetilde{L_{f}^{(A)}}(z)=\int_{\Omega} f(x) \widetilde{A}\left(g_{z}(x)\right) d \mu(x) .
$$

The last integral is the definition of convolution (in $G$ ) of $f$ and $\widetilde{A}[\underline{H}]$ :

$$
\widetilde{L_{f}^{(A)}}=f * \widetilde{A} \text {. }
$$

As $A=P_{\mathbf{m}}$ we have

$$
\begin{aligned}
\widetilde{A}(z) & =\left\langle P_{\mathbf{m}} k_{z}, k_{z}\right\rangle=K(z, z)^{-1}\left(P_{\mathbf{m}} K_{z}\right)(z) \\
& =K(z, z)^{-1} K_{\mathbf{m}}(z, z),
\end{aligned}
$$


where $K_{\mathbf{m}}(x, y)$ is the reproducing kernel of the subspace $\mathcal{P}_{\mathbf{m}} \subset \mathcal{H}$. In particular, for $\mathbf{m}=(0, \ldots, 0)$, we have $\widetilde{P_{(0, \ldots, 0)}}(z)=K(z, z)^{-1}$.

Now it was shown by Ørsted and Zhang [OZ, Proposition 3.15, that there exists a unique $G$-invariant linear partial differential operator $D=D^{\mathrm{m}}$ on $\Omega$ such that

$$
D K(z, z)^{-1}=K_{\mathbf{m}}(z, z) K(z, z)^{-1} .
$$

Arguing as in the Fock-space case, it follows that

$$
\begin{aligned}
\widetilde{L_{f}^{(A)}} & =f * \widetilde{P_{\mathbf{m}}}=f * D \widetilde{P_{(0, \ldots, 0)}} \\
& =D f * \widetilde{P_{(0, \ldots, 0)}} \\
& =\widetilde{T_{D f}},
\end{aligned}
$$

whence $L_{f}^{(A)}=T_{D f}$ by part (b) of Proposition 3, This completes the proof.

Remark. Again, it is evident from the proof that (28) holds not only for $f \in \mathcal{D}(\Omega)$, but for any $f \in C^{\infty}(\Omega)$ whose derivatives do not grow too fast at the boundary, so that the partial integration implicit in the third equality in (28) is legitimate.

\section{REFERENCES}

[Ar] J. Arazy: A survey of invariant Hilbert spaces of analytic functions on bounded symmetric domains, Multivariable operator theory (R.E. Curto, R.G. Douglas, J.D. Pincus, N. Salinas, editors), Contemporary Mathematics 185, Amer. Math. Soc., Providence, 1995, pp. 7-65. MR $1332053(96 \mathrm{e}: 46034)$

[AU] J. Arazy, H. Upmeier: Invariant symbolic calculi and eigenvalues of invariant operators on symmetric domains, Function spaces, interpolation theory, and related topics (Lund, 2000) (A. Kufner, M. Cwikel, M. Engliš, L.-E. Persson, G. Sparr, editors), Walter de Gruyter, Berlin, 2002, pp. 151-211. MR1943284 (2003k:32031)

[BC1] C.A. Berger, L.A. Coburn: Toeplitz operators and quantum mechanics, J. Funct. Anal. 68 (1986), 273-299. MR859136 (88b:46098)

[BC2] C.A. Berger, L.A. Coburn: Toeplitz operators on the Segal-Bargmann space, Trans. Amer. Math. Soc. 301 (1987), 813-829. MR882716 (88c:47044)

[BC3] C.A. Berger, L.A. Coburn: Heat flow and Berezin-Toeplitz estimates, Amer. J. Math. 116 (1994), 563-590. MR.1277446 (95g:47038)

[Be] F.A. Berezin: Covariant and contravariant symbols of operators, Izv. Akad. Nauk SSSR Ser. Mat. 36 (1972), 1134-1167 (in Russian); English translation in Math. USSR Izv. 6 (1972), 1117-1151. MR0350504 (50:2996)

[C1] L. Coburn: On the Berezin-Toeplitz calculus, Proc. Amer. Math. Soc. 129 (2001), 33313338. MR1845010 (2002e:47031)

[C2] L. Coburn: The Bargmann isometry and Gabor-Daubechies wavelet localization operators, Systems, approximation, singular integral operators, and related topics (A. Borichev, N. Nikolski, editors), Operator Theory: Advances and Applications 129, Birkhäuser Verlag, Basel, 2001, pp. 169-178. MR1882695 (2003a:47054)

[C3] L. Coburn: Symbol calculus for Gabor-Daubechies windowed Fourier localization operators, preprint.

[D1] I. Daubechies: Time frequency localization operators: A geometric phase space approach, IEEE Trans. Inform. Theory 34 (1988), 605-612. MR966733

[D2] I. Daubechies: Ten lectures on wavelets, CBMS-NSF Regional Conference Series 6, SIAM, Philadelphia, 1992. MR.1162107 (93e:42045)

[E] M. Engliš: Toeplitz operators and group representations, J. Fourier Anal. Appl., 13 (2007), 243-265. MR 2334609 (2008e:47072)

[FN] H.G. Feichtinger, K. Nowak: A Szegö-type theorem for Gabor-Toeplitz localization operators, Michigan Math. J. 49 (2001), 13-21. MR.1827072 (2003b:47049)

[Fo] G.B. Folland: Harmonic analysis in phase space, Annals of Mathematics Studies 122, Princeton University Press, Princeton, 1989. MR.983366 (92k:22017) 
[H] S. Helgason: Groups and geometric analysis, Academic Press, Orlando, 1984. MR754767 (86c:22017)

[Ja] J. Janas: Unbounded Toeplitz operators in the Segal-Bargmann space III, Math. Scand. 88 (2001), 126-136. MR1813524 (2002a:47043)

[Ko] A. Korányi: Function spaces on bounded symmetric domains, Analysis and geometry on complex homogeneous domains (J. Faraut, S. Kaneyuki, A. Korányi, Q.-K. Lu, G. Roos, editors), Progress in Mathematics 185, Birkhäuser, Basel-Boston-Berlin, 2000.

[Lo] M.-L. Lo: The Bargmann transform and windowed Fourier localization, Integral Equations Operator Theory 57 (2007), 397-412. MR2307818(2008b:47049)

[MN] F. de Mari, K. Nowak: Localization type Berezin-Toeplitz operators on bounded symmetric domains, J. Geom. Anal. 12 (2002), 9-27. MR1881289 (2003b:47048)

[OZ] B. Ørsted, G. Zhang: Weyl quantization and tensor products of Fock and Bergman spaces, Indiana Univ. Math. J. 43 (1994), 551-583. MR1291529 (95h:22008)

[Ru] W. Rudin: Functional analysis, McGraw-Hill, 1973. MR.0365062 (51:1315)

[Up] H. Upmeier: Toeplitz Operators and Index Theory in Several Complex Variables, Operator Theory: Advances and Applications 81, Birkhäuser, Basel, 1996. MR.1384981 (97f:47022)

[Wo] M.W. Wong: Wavelet transforms and localization operators, Operator Theory: Advances and Applications 136, Birkhäuser Verlag, Basel, 2002. MR1918652 (2003i:42003)

[Zh] K. Zhu: Operator theory in function spaces, Marcel Dekker, New York, 1990. MR.1074007 (92c:47031)

Mathematics Institute, Silesian University at Opava, Na RybníčKu 1, 74601 Opava, Czech Republic - and - Mathematics Institute, Žitná 25, 11567 Prague 1, Czech Republic

E-mail address: englis@math.cas.cz 